\title{
Age-related low skeletal muscle mass correlates with joint space narrowing in knee osteoarthritis in a South Korean population: a cross-sectional, case-control study
}

\author{
Hyun-Je Kim ${ }^{1}$, Young-Hoon Hong ${ }^{2}$ \\ ${ }^{1}$ Division of Rheumatology, Department of Internal Medicine, CHA University, CHA Gumi Medical Center, Gumi, Korea \\ ${ }^{2}$ Division of Rheumatology, Department of Internal Medicine, Yeungnam University College of Medicine, Daegu, Korea
}

Background: This study was conducted to analyze the effects of low skeletal muscle mass index (SMI) and obesity on aging-related osteoarthritis (OA) in the Korean population.

Methods: A total of 16,601 participants who underwent a dual-energy X-ray absorptiometry and 3,976 subjects with knee X-rays according to the modified Kellgren-Lawrence $(K L)$ system were enrolled. Knees of $\geq K L$ grade 2 were classified as radiologic OA. The severity of joint space narrowing (JSN) was classified by X-rays as normal, mild-to-moderate, and severe JSN in radiologic OA. The subjects were grouped as normal SMI (SMI of $\geq-1$ standard deviation [SD] of the mean), low SMI class I (SMI of $\geq-2$ SDs and $<-1$ SD), and low SMI class II (SMI of $<-2$ SDs). Obesity was defined as a body mass index (BMI) of $\geq 27.5 \mathrm{~kg} / \mathrm{m}^{2}$.

Results: The modified KL grade and JSN severity were negatively correlated with the SMI and positively correlated with BMI and age. The SMI was negatively correlated with age. JSN severity was significantly associated with a low SMI class compared to a normal SMI, which was more prominent in low SMI class II than class I. Obesity was significantly associated with more severe JSN, only for obesity with a low SMI class. Furthermore, patients with a low SMI class, regardless of obesity, were prone to having more severe JSN.

Conclusion: This study suggested that a low SMI class was associated with aging and that an age-related low SMI was more critically related to the severity of JSN in OA.

Keywords: Body composition; Body mass index; Obesity; Osteoarthritis; Sarcopenia

\section{Introduction}

The age-related degenerative loss of skeletal muscle mass (SMM) and strength is referred to as sarcopenia, which can accompany obesity as sarcopenic obesity [1]. Baumgartner et al. [2] defined sarcopenia as an appendicular SMM (ASM) divided by body height squared in meters that is less than two standard deviations (SDs) below the reference values for young and healthy individu- als of the same sex and ethnicity. However, height loss related to aging changes in the bones, muscles, and joints [3] can be a factor in increasing the height-adjusted SMM index $\left(\mathrm{SMI}\left[\mathrm{kg} / \mathrm{m}^{2}\right]\right)$ despite changes in the ASM. Janssen et al. [4] proposed percentage-SMI which was the absolute SMM $(\mathrm{kg})$ to weight percentage (muscle mass/body mass $\times 100$ ), and defined sarcopenia as an SMI of less than one SD below the bioelectrical impedance analysis (BIA) reference values measured in sex- and ethnicity-matched healthy indi-

Received: October 7, $2021 \cdot$ Revised: December 23, 2021 • Accepted: December 24, 2021

Corresponding author: Young-Hoon Hong, MD, PhD

Division of Rheumatology, Department of Internal Medicine, Yeungnam University College of Medicine, 170 Hyeonchung-ro, Nam-gu, Daegu 42415, Korea

Tel: +82-53-620-3841・Fax: +82-53-654-8386•E-mail: yhhong@med.yu.ac.kr 
viduals between the ages of 18 and 39 years. An SMI value between -1 SD and -2 SDs of the mean value is referred to as class I sarcopenia, and an SMI value below -2 SDs of the mean value as class II sarcopenia. However, recently defining sarcopenia has required impaired skeletal muscle strength in addition to the loss of SMM $[5,6]$. To measure body composition, dual-energy X-ray absorptiometry (DEXA) is the standard for clinical analysis [7]. In this study, DEXA-measured SMM and SMI were evaluated according to the criteria proposed by Janssen et al. [4]. And different from sarcopenia, an SMI value between -1 SD and -2 SDs of the mean value was designated as age-related low SMI class I, and an SMI value below -2 SDs of the mean value as age-related low SMI class II.

Obesity causes medical complications and deteriorates the quality of life. In general, the body mass index (BMI), a measure of body fat by dividing weight by height, is used for determining obesity in adults [8]. According to the World Health Organization criteria, a BMI between 18.5 and $24.9 \mathrm{~kg} / \mathrm{m}^{2}$ is defined as normal [9]; a BMI of higher than $30 \mathrm{~kg} / \mathrm{m}^{2}$ is known to increase the risk of cardiovascular disease, stroke, osteoarthritis (OA), and increase mortality [10]. In the Asian population, a BMI between 18.5 and $23.5 \mathrm{~kg} / \mathrm{m}^{2}$ is defined as normal and a BMI of greater than $27.5 \mathrm{~kg} / \mathrm{m}^{2}$ is associated with a higher risk of diabetes and cardiovascular disease [11].

Primary OA is an age-related disorder characterized by degradation of the articular cartilage and the substantial loss of matrix [12]. The symptoms and signs gradually develop after the fifth decade [13], and approximately $68 \%$ of females and $58 \%$ of males older than 65 years of age have OA [14]. There are several contributing factors to the development of $\mathrm{OA}$, such as cartilage matrix degradation and intraarticular cell senescence $[15,16]$, extraarticular loss of SMM [17], and deterioration of proprioception [18]. In addition to age, obesity is associated with an increased risk of knee OA, especially in older subjects $[14,19]$. The American College of Rheumatology (ACR) criteria for OA of the knee consists of age, joint symptoms, the lack of inflammatory conditions, and positive radiography of the knee [20]. The Kellgren-Lawrence (KL) system has been used for the radiographic grading of knee OA according to the presence and severity of osteophytosis, joint space narrowing (JSN), bony sclerosis, and subchondral cyst formation [21].

As body composition changes with increasing fat and decreasing SMM with aging [22], the authors hypothesized that low SMM and SMI values would be parameters for disordered body composition, and age-related low SMI would be critically associated with OA severity as an aging process. However, since there have been a few studies investigated the relationship between age-related low
SMI, OA, and JSN, this study was conducted to analyze changes in the SMI values with aging and the significances of an age-related low SMI on OA severity using nationwide data surveyed for 4 years in South Korean population.

\section{Methods}

Ethical statements: This study was performed in accordance with the 1964 Declaration of Helsinki. The study protocol for performing an analysis of the KNHANES data (IV-2, 3 and V-1, 2; January 2008-December 2011) was approved by the Institutional Review Board (IRB) of the Korea Centers for Disease Control and Prevention (IRB No: 2011-02CON06-C). Informed consent was obtained from all participants when the KNHANES was conducted.

\section{Study populations and data}

The subjects and data in this study were obtained from the 4th and 5th Korean National Health and Nutrition Examination Survey (KNHANES IV-2, 3 and V-1, 2; January 2008-December 2011). The KNHANES is a nationwide, cross-sectional survey representing the South Korean population conducted by the Korea Centers for Disease Control and Prevention. In stratified, multistage, probability, independent rolling survey sampling, health interview survey, health examination, and nutrition survey were carried out annually in the target population of noninstitutionalized civilians older than 1 year of age in South Korea. The sampling units were based on geographic area, home size, house price, sex, and age group according to the household registries from the National Census Registry and the current quotation of apartment. A total of 37,753 participants $(9,744$ individuals, 3,707 households and 200 national districts in 2008; 10,533, 3,975, and 200 in 2009; 8,958, 3,278, and 192 in 2010; and 8,518, 3,289, and 192 in 2011) were included in the survey and provided informed consent. Of these, males were 17,195 and females were 20,558. Anthropometric measurements, body composition, blood tests, and self-reported health status including medical and social history were available as data. Regarding the musculoskeletal system, lateral view radiographs of bilateral knees were taken from all the participants aged 50 years and older standing in the anteroposterior position with $30^{\circ}$ of flexion. DEXA were performed to measure the body composition in those aged 10 years and older. Among the participants providing informed consent, those who were definitely or possibly pregnant, incapable of lying in the supine position, weighing over $159 \mathrm{~kg}$, or with an injection of a radiocontrast agent within 7 days or radioisotopes in the previous 3 days were excluded. Based on 
the KNHANES data, a total of 16,601 participants $(7,188$ males and 9,413 females) who underwent a DEXA, including 5,929 healthy subjects (2,572 males, 3,357 females) aged between 18 and 39 years and 3,976 subjects with knee radiographs graded on the $\mathrm{KL}$ system were enrolled in the study. Any person with an erythrocyte sedimentation rate of $\geq 40 \mathrm{~mm} / \mathrm{hr}$, positive rheumatoid factor, abnormal laboratory tests or chest $\mathrm{X}$-ray results, or a medical history of diabetes, malignancy, joint trauma or surgery, or other metabolic or inflammatory diseases were excluded from the study.

\section{Determination of body mass and composition by dual- energy X-ray absorptiometry}

ASM, SMI, and BMI were determined on the basis of the DEXA. The data were acquired using a Discovery-W scanner (Hologic, Marlborough, MA, USA) in standardized equipment and procedures. ASM was defined as the sum of the lean mass of the arms and legs minus the bone mineral content and determined according to the method of Heymsfield et al. [23]. In this study, SMI was defined as the absolute ASM to the percentage of body mass (\%) [7], and BMI as weight $/$ height ${ }^{2}\left(\mathrm{~kg} / \mathrm{m}^{2}\right)$. Low SMI was defined as SMI below -1 SD of the mean SMI measured from sex-matched healthy individuals between the ages of 18 and 39 years, which was subdivided into class I and class II. The BMI cutoff values were $<18.5 \mathrm{~kg} / \mathrm{m}^{2}$ for underweight, 18.5 to $<23 \mathrm{~kg} / \mathrm{m}^{2}$ for the normal body weight (BW), 23 to $<27.5 \mathrm{~kg} / \mathrm{m}^{2}$ for overweight, and $\geq 27.5$ $\mathrm{kg} / \mathrm{m}^{2}$ for obese, as suggested for the Asian population [11]. The DEXA data were assessed to compare the effects of low SMI, and obesity on JSN severity in age-related OA in the population. The effects of obesity on OA-JSN were further analyzed according to SMI status (normal SMI, low SMI class I, and low SMI class II) in contrast to other studies $[5,7,24]$.

\section{Radiologic osteoarthritis, Kellgren-Lawrence grade, and the severity of joint space narrowing of the knee}

Regarding OA, the anteroposterior and lateral view knee X-rays views were taken in the target population aged over 50 years using a SD3000 Synchro Stand (Accele Ray; Shinyoung, Seoul, Korea). Subsequently, the presence and severity of bony changes in OA were graded by two experts in musculoskeletal radiology using the KL grading system [21]. No abnormal radiological findings were designated as grade 0 ; mild degenerative changes in both knees accompanying possible osteophytes without enthesophytes as grade 1; degenerative $\mathrm{OA}$ in both knees with definite osteophytes but no JSN as grade 2; degenerative OA in both knees with definite osteophytes and possible JSN up to moderate degree as grade 3 ; and degenerative OA in both knees with subchondral sclerosis and JSN beyond grade 3 up to severe degree as grade $4[21,25]$. Knees showing a $\mathrm{KL}$ grade of $\geq 2$ were classified as having knee radiologic OA (ROA), consistent with the ACR criteria $[20,21]$. In case without concordance in the grade between the two radiologists, the higher grade was accepted. However, if the discrepancy was greater than 1 grade, the third radiologist was consulted, and the grade concordant with the third grade, or the grade of the first radiologist was accepted (interrater agreement within 1 grade of difference: $92.8 \%$ for KNHANES IV-2 and 3, and $95.2 \%$ for KNHANES V-1 and 2; weighted Cohen's kappa coefficients of 0.65 and 0.74 , respectively). The knees with radiological findings of secondary causes and joint replacement were excluded from grading. Finally, the higher of the grades of both knees was accepted as the $\mathrm{KL}$ grade of the subject. Among the patients with ROA, KL grade 2 was categorized as no JSN according to the definition, $\mathrm{KL}$ grade 3 as mild-to-moderate JSN, and KL grade 4 as severe JSN. The radiologic grades and the severity of ROA-JSN were analyzed according to the SMI, BMI, and SMI classifications stratified according to a BMI of obesity.

\section{Statistical analyses}

The values are expressed as the mean \pm SD. DEXA measurements were compared using the independent Student $t$-test and the anal$y$ sis of variance test. Analysis of correlations between the determinants was performed using Pearson correlation test and a cross-tab with the chi-square test and adjusted using linear and logistic regression tests. Cohen's kappa test was used to determine intraobserver variation. The results were considered statistically significant for $p$-values of $<0.05$ in the $95 \%$ confidence interval (CI) and $p$-values of $<0.001$ in the $99 \%$ CI. Statistical analyses were performed using SPSS program ver. 12 (SPSS Inc., Chicago, IL, USA).

\section{Results}

The mean SMI value was $33.17 \pm 2.97$ in healthy males $(\mathrm{n}=2,572)$ and $26.23 \pm 2.48$ in healthy females $(n=3,357)$ aged 18 to 39 years. In the reference population, the SMI and BMI values were lower in females than in males. In males, SMI values greater than 30.20 , between 30.20 and 27.23, and less than 27.23 were designated as normal, low SMI class I, and low SMI class II, respectively. SMI values greater than 23.75 , between 23.75 and 21.27 , and less than 21.27 were designated as normal, low SMI class I, and low SMI class II, respectively, in females based on the reference values (Table 1).

ASM and SMI decreased gradually throughout all ages after the second decade $(p<0.01)$ (Table 2). BW showed a steady increase from the second decade to the fifth decade of age, after then, shift- 
ed to a gradual decrease $(p<0.01)$. Age was negatively correlated with ASM $(r=-0.137)$ and SMI $(r=-0.172)$, and significantly positively correlated with BMI $(r=0.182)$. Since the sixth decade of age, ASM, and SMI demonstrated a consistent decrease

Table 1. Reference values for the classification of age-related low SMI loss in a South Korean population

\begin{tabular}{lcc}
\hline Variable & $\mathrm{BMI}^{\mathrm{b})}\left(\mathrm{kg} / \mathrm{m}^{2}\right)$ & $\mathrm{SMI}^{\mathrm{b})}(\%)$ \\
\hline Male $^{\mathrm{a})}(\mathrm{n}=2,572)$ & $23.94 \pm 3.45$ & $33.17 \pm 2.97$ \\
Low SMI loss class I & & $27.23,<30.20$ \\
Low SMI loss class II & & $<27.23$ \\
Female $^{\text {a) }}(\mathrm{n}=3,357)$ & $21.94 \pm 3.46$ & $26.23 \pm 2.48$ \\
Low SMI loss class I & $\geq 21.27,<23.75$ \\
Low SMI loss class II & & $<21.27$ \\
\hline
\end{tabular}

Values are presented as mean \pm standard deviation.

$\mathrm{SMI}$, skeletal muscle mass index; BMI, body mass index.

${ }^{\text {a) }}$ Aged between $\geq 18$ and $\leq 39$ years. ${ }^{b}$ Measured using dual-energy $X$-ray absorptiometry. $(p<0.01)$ (Table 3). The results suggest that low SMI values reflect age-related loss and that age-related SMI decreases become critical after the sixth decade of age.

The modified KL grade and JSN grade also had negative correlations with ASM $(r=-0.162$ and $r=-0.264$, respectively $)$ and SMI values $(r=-0.211$ and $r=-0.271$, respectively), whereas they were positively correlated with BMI $(r=0.19$ and $r=0.118$, respectively) and age $(r=0.418$ and $r=0.248$, respectively) (Table 2$)$. The modified KL grade and JSN showed a positive linear relationship with age in the Korean population aged $\geq 50$ years $(r=0.418$, $p<0.01$ and $\mathrm{r}=0.248, p<0.01$, respectively).

As for the relationship of SMI, age, and BMI with severity of JSN, the odds ratios (ORs) for mild-to-moderate JSN $(n=625)$ and severe JSN $(n=351)$ were evaluated compared to the no JSN group $(\mathrm{n}=576)$. A lower SMI value (OR, 0.900 [95\% CI, 0.8730.929] for mild-to-moderate JSN; OR, 0.836 [95\% CI, $0.802-$ 0.872 ] for severe JSN), higher age (OR, 1.041 [95\% CI, 1.026-

Table 2. Comparison of body mass and composition according to the decades of age, Kellgren-Lawrence grade, and radiologic osteoarthritis of the knee grades

\begin{tabular}{|c|c|c|c|c|c|}
\hline Variable & No. of subjects & ASM (kg) & SMI (\%) & BMI $\left(\mathrm{kg} / \mathrm{m}^{2}\right)$ & Age (yr) \\
\hline \multicolumn{6}{|l|}{ Age (yr) } \\
\hline $10-19$ & 1,174 & $16.67 \pm 5.06$ & $30.37 \pm 4.50$ & $20.71 \pm 3.69$ & NA \\
\hline $30-39$ & 3,495 & $18.58 \pm 5.14$ & $28.94 \pm 4.20$ & $23.14 \pm 3.46$ & NA \\
\hline $40-49$ & 3,297 & $18.35 \pm 4.79$ & $28.47 \pm 4.17$ & $23.87 \pm 3.20$ & NA \\
\hline $50-59$ & 2,708 & $17.54 \pm 4.34$ & $27.88 \pm 4.28$ & $24.08 \pm 2.89$ & NA \\
\hline $70-79$ & 1,398 & $15.72 \pm 3.69$ & $27.70 \pm 4.36$ & $23.29 \pm 3.29$ & NA \\
\hline $80-92$ & 312 & $14.31 \pm 3.26$ & $27.42 \pm 3.75$ & $22.43 \pm 2.89$ & NA \\
\hline Total & 16,601 & $17.75 \pm 4.82$ & $28.59 \pm 4.36$ & $23.29 \pm 3.42$ & NA \\
\hline r-value & & $-0.137^{* *}$ & $-0.172^{* *}$ & $0.182^{* *}$ & NA \\
\hline \multicolumn{6}{|c|}{ Kellgren-Lawrence grade } \\
\hline 3 & 625 & $15.81 \pm 3.74$ & $26.26 \pm 4.03$ & $24.70 \pm 3.20$ & $67.18 \pm 8.87$ \\
\hline 4 & 351 & $14.60 \pm 3.22$ & $25.23 \pm 3.78$ & $24.93 \pm 3.66$ & $70.92 \pm 8.19$ \\
\hline Total & 3,976 & & & & \\
\hline r-value & & $-0.162^{* *}$ & $-0.211^{* *}$ & $0.19^{* *}$ & $0.418^{* *}$ \\
\hline \multicolumn{6}{|l|}{ JSN grade } \\
\hline No JSN & 576 & $17.29 \pm 4.11$ & $28.14 \pm 4.20$ & $23.98 \pm 2.82$ & $64.90 \pm 8.80$ \\
\hline Mild-to-moderate & 625 & $15.81 \pm 3.74$ & $26.26 \pm 4.03$ & $24.70 \pm 3.20$ & $67.18 \pm 8.87$ \\
\hline Severe & 351 & $14.60 \pm 3.22$ & $25.23 \pm 3.78$ & $24.93 \pm 3.66$ & $70.92 \pm 8.19$ \\
\hline Total & 1,552 & $16.09 \pm 3.91$ & $26.72 \pm 4.20$ & $24.48 \pm 3.20$ & $67.18 \pm 8.98$ \\
\hline r-value & & $-0.264^{* *}$ & $-0.271^{* *}$ & $0.118^{* *}$ & $0.248^{* *}$ \\
\hline
\end{tabular}

Values are presented as mean \pm standard deviation.

ASM, appendicular skeletal muscle mass; SMI, skeletal muscle mass index; BMI, body mass index; NA, not applicable; JSN, joint space narrowing. ${ }^{* *} p<0.01$. 
1.055] for mild-to-moderate JSN; OR, 1.099 [95\% CI, 1.0801.118 ] for severe JSN) and a higher BMI (OR, 1.047 [95\% CI, 1.003-1.093] for mild-to-moderate JSN; OR, 1.067 [95\% CI, 1.013-1.124] for severe JSN) were significantly associated with JSN in knee ROA (Table 3).

The effect of BMI on the severity of JSN was evaluated with a multinomial logistic regression analysis. The group with obesity had as significantly higher association with mild-to-moderate JSN (OR, 2.174; 95\% CI, 1.501-3.149) and severe JSN (OR, 2.307; 95\% CI, 1.527-3.484) in knee ROA, which was more prominent in females. However, in the group with obesity, the mean BMI values according to the severity of JSN were not statistically different (Table 4).

This study investigated the significance of a low SMI on the severity of JSN according to the low SMI classes using multivariate regression analysis. The results demonstrated that low SMI values such as age-related SMI loss were significantly associated with mild-to-moderate JSN (OR, 1.335 [95\% CI, 1.031-1.727] in class I; OR, 1.696 [95\% CI, 1.076-2.674] in class II), and severe JSN (OR, 1.442 [95\% CI, 1.065-1.953] in class I; OR, 2.916 [95\% CI, $1.814-4.689$ ] in class II) in Korean aged $\geq 50$ years with knee ROA (Table 5). These results suggest that the Korean population with greater age-related SMI loss tended to have more severe JSN in knee ROA. The mean SMI values in the no-JSN group were higher than those with mild-to-moderate JSN and severe JSN (Table 5).

As described above, BMI-obesity was significantly associated with the severity of JSN. However, when the effect was analyzed according to the presence and degree of low SMI values, this study revealed that even though the subjects were obese, the effect of

Table 3. Relationship between SMI and JSN in radiologic osteoarthritis

\begin{tabular}{lcccc}
\hline JSN grade & No. of subjects (M/F) & & $\beta$ & OR (95\% Cl) \\
\hline No JSN & $576(272 / 304)$ & & & $0.900(0.873-0.929)^{* *}$ \\
Mild-to-moderate JSN & $625(162 / 463)$ & SMI & -0.105 & $1.041(1.026-1.055)^{* *}$ \\
& & Age & 0.040 & $1.047(1.003-1.093)^{*}$ \\
Severe JSN & & $\mathrm{BMI}$ & 0.046 & $0.836(0.802-0.872)^{* *}$ \\
& & $\mathrm{SMl}$ & -0.179 & $1.099(1.080-1.118)^{* *}$ \\
\end{tabular}

$\mathrm{SMI}$, skeletal muscle mass index; JSN, joint space narrowing; $\mathrm{M}$, male; $\mathrm{F}$, female; $\mathrm{OR}$, odds ratio; $\mathrm{Cl}$, confidence interval; $\mathrm{BMI}$, body mass index. ORs and $95 \% \mathrm{Cls}$ were determined by multivariate analysis with adjustments for $\mathrm{SMI}$, age, and $\mathrm{BMI}$.

${ }^{*} p<0.05,{ }^{* *} p<0.01$

Table 4. JSN adjusted by BMI class for total radiologic osteoarthritis population

\begin{tabular}{|c|c|c|c|c|}
\hline \multirow{2}{*}{ JSN grade } & \multicolumn{4}{|c|}{ BMI } \\
\hline & Normal & Underweight* & Overweight* & Obesity* \\
\hline \multicolumn{5}{|l|}{ No JSN } \\
\hline No. of subjects & 201 & 10 & 303 & 62 \\
\hline $\mathrm{BMI}^{\mathrm{a})}\left(\mathrm{kg} / \mathrm{m}^{2}\right)$ & $21.27 \pm 1.23$ & $17.49 \pm 0.78$ & $24.98 \pm 1.21$ & $28.97 \pm 1.24$ \\
\hline \multicolumn{5}{|l|}{ Mild-to-moderate } \\
\hline No. of subjects & 170 & 15 & 326 & 114 \\
\hline $\mathrm{BM}^{\mathrm{a})}\left(\mathrm{kg} / \mathrm{m}^{2}\right)$ & $21.39 \pm 1.18$ & $18.03 \pm 0.40$ & $25.04 \pm 1.23$ & $29.53 \pm 1.95$ \\
\hline$\beta$ & & 0.573 & 0.241 & $0.777^{* *}$ \\
\hline OR $(95 \% \mathrm{Cl})$ & & $1.774(0.777-4.05)$ & $1.272(0.984-1.645)$ & $2.174(1.501-3.149)$ \\
\hline \multicolumn{5}{|l|}{ Severe } \\
\hline No. of subjects & 104 & 7 & 166 & 74 \\
\hline $\mathrm{BM}^{\mathrm{a})}$ & $21.28 \pm 1.17$ & $17.15 \pm 0.87$ & $25.29 \pm 1.21$ & $29.99 \pm 2.82$ \\
\hline$\beta$ & & 0.302 & 0.057 & $0.836^{* *}$ \\
\hline OR $(95 \% \mathrm{Cl})$ & & $1.353(0.5-3.657)$ & $1.059(0.782-1.434)$ & $2.307(1.527-3.484)$ \\
\hline
\end{tabular}

JSN, joint space narrowing; $\mathrm{BMI}$, body mass index; $\mathrm{OR}$, odds ratio; $\mathrm{Cl}$, confidence interval.

${ }^{\text {a) }}$ Values are presented as mean \pm standard deviation.

Within the class, analysis of variance was performed; ${ }^{*} p<0.05,{ }^{* *} p<0.01$. 
obesity on the severity of JSN was not consistent in the subgroup with a normal. Furthermore, in higher classes of low SMI, the BMI-obesity effect on the severity of JSN was greater (OR, 1.852 [95\% CI, 1.153-2.976] for mild-to-moderate JSN and OR, 2.732 [95\% CI, 1.648-4.527] for severe JSN in low SMI class I vs. OR, 2.361 [95\% CI, 1.173-4.751] for mild-to-moderate JSN and OR, 3.415 [95\% CI, 1.634-7.136] for severe JSN in low SMI class II) (Table 6). The results may suggest that the presence and degree of low SMI values are critical to ROA-JSN in obese patients.

\section{Discussion}

It is natural for SMM to diminish after the adolescent period, and the decrease can be as much as $0.5 \%$ to $1 \%$ per year after 25 years of age [26]. The loss of SMM, quality, and strength in association with advancing age referred to as sarcopenia, which is a degenerative process. Rosenberg [27] first coined the term "sarcopenia" in 1989, to describe the age-associated loss of SMM. Baumgartner et al. [2] proposed the widely used definitions of sarcopenia and sar-

Table 5. Comparing mean values of SMI in JSN classes adjusted by SMI class for total radiologic osteoarthritis population

\begin{tabular}{|c|c|c|c|}
\hline \multirow{2}{*}{ JSN grade } & \multicolumn{3}{|c|}{ SMI } \\
\hline & Normal $^{* *}$ & Low SMI class I* $^{* *}$ & Low SMI class I** \\
\hline \multicolumn{4}{|l|}{ No JSN } \\
\hline No. of subjects & 397 & 146 & 33 \\
\hline $\mathrm{SMI}^{\mathrm{a})}(\%)$ & $29.69 \pm 3.66$ & $25.02 \pm 3.09$ & $23.36 \pm 3.04$ \\
\hline \multicolumn{4}{|l|}{ Mild-to-moderate } \\
\hline No. of subjects & 383 & 188 & 54 \\
\hline $\mathrm{SMI}^{\mathrm{a})}(\%)$ & $27.99 \pm 3.65$ & $24.19 \pm 2.74$ & $21.08 \pm 2.14$ \\
\hline$\beta$ & & $0.289^{*}$ & $0.528^{*}$ \\
\hline OR $(95 \% \mathrm{Cl})$ & & $1.335(1.031-1.727)$ & $1.696(1.076-2.674)$ \\
\hline \multicolumn{4}{|l|}{ Severe } \\
\hline No. of subjects & 198 & 105 & 48 \\
\hline $\mathrm{SMI}^{\mathrm{a})}(\%)$ & $27.33 \pm 3.15$ & $23.39 \pm 2.39$ & $20.55 \pm 1.90$ \\
\hline$\beta$ & & $0.366^{*}$ & $1.07^{* *}$ \\
\hline OR $(95 \% \mathrm{Cl})$ & & $1.442(1.065-1.953)$ & 2.916 (1.814-4.689) \\
\hline
\end{tabular}

$\mathrm{SMI}$, skeletal muscle mass index; JSN, joint space narrowing; $\mathrm{OR}$, odds ratio; $\mathrm{Cl}$, confidence interval.

${ }^{a}$ Values are presented as mean \pm standard deviation.

Within class, analysis of variance was performed; ${ }^{*} p<0.05,{ }^{* *} p<0.01$.

Table 6. Comparing mean values of SMI in JSN adjusted by SMI and BMI classification for total radiologic osteoarthritis population

\begin{tabular}{|c|c|c|c|c|c|c|}
\hline \multirow{3}{*}{ JSN grade } & \multicolumn{6}{|c|}{ BMI } \\
\hline & \multicolumn{3}{|c|}{ Non-obese } & \multicolumn{3}{|c|}{ Obese } \\
\hline & Normal SMI** & Low SMI class I* & Low SMI class II* & Normal SM"** & Low SMI class I* & Low SMI class II* \\
\hline \multicolumn{7}{|l|}{ No JSN } \\
\hline No. of subjects & 377 & 116 & 21 & 20 & 30 & 12 \\
\hline $\mathrm{SMI}^{\mathrm{a})}(\%)$ & $29.71 \pm 3.67$ & $25.10 \pm 3.14$ & $23.22 \pm 2.96$ & $29.26 \pm 3.60$ & $24.69 \pm 2.93$ & $23.59 \pm 3.29$ \\
\hline \multicolumn{7}{|l|}{ Mild-to-moderate } \\
\hline No. of subjects & 346 & 137 & 28 & 37 & 51 & 26 \\
\hline $\mathrm{SMI}^{\mathrm{a})}(\%)$ & $28.11 \pm 3.67$ & $24.53 \pm 2.81$ & $21.45 \pm 2.33$ & $26.95 \pm 3.37$ & $23.27 \pm 2.32$ & $20.67 \pm 1.88$ \\
\hline$\beta$ & & 0.252 & 0.373 & $0.701^{*}$ & $0.616^{*}$ & $0.859^{*}$ \\
\hline OR $(95 \% \mathrm{Cl})$ & & $1.287(0.966-1.715)$ & $1.453(0.81-2.606)$ & $2.016(1.148-3.54)$ & $1.852(1.153-2.976)$ & $2.361(1.173-4.751)$ \\
\hline \multicolumn{7}{|l|}{ Severe } \\
\hline No. of subjects & 184 & 65 & 28 & 14 & 40 & 20 \\
\hline $\mathrm{SMI}^{\mathrm{a})}(\%)$ & $27.44 \pm 3.16$ & $23.80 \pm 2.60$ & $20.87 \pm 1.88$ & $25.90 \pm 2.71$ & $22.73 \pm 1.71$ & $20.10 \pm 1.87$ \\
\hline$\beta$ & & 0.138 & $1.005^{* *}$ & 0.361 & $1.005^{* *}$ & $1.228^{* *}$ \\
\hline OR $(95 \% \mathrm{Cl})$ & & $1.148(0.808-1.631)$ & $2.732(1.51-4.941)$ & $1.434(0.708-2.904)$ & $2.732(1.648-4.527)$ & $3.415(1.634-7.136)$ \\
\hline
\end{tabular}

SMI, skeletal muscle mass index; JSN, joint space narrowing; $\mathrm{BMI}$, body mass index; OR, odds ratio; $\mathrm{Cl}$, confidence interval.

${ }^{a}$ Values are presented as mean \pm standard deviation.

Within class, analysis of variance was performed; ${ }^{*} p<0.05,{ }^{* *} p<0.01$. 
copenic obesity. Age-related alterations in body composition are the combination of reduced muscle mass and excess fat mass despite the prevalence of obesity, as sarcopenia or sarcopenic obesity [28].

$\mathrm{BMI}$, the ratio between weight and height, representing body fat, is the most commonly used measure of obesity, and in cross-sectional studies in large populations, BMI values have indicated that the incidence of obesity is increasing in older people. However, after reaching its peak at 50 to 60 years of age, it tends to decline in both sexes [14,29].

The mean SMI value in the young and healthy South Korean population was $33.17 \pm 2.97$ in males and $26.23 \pm 2.48$ in females. The mean and cutoff values for classifying low SMM were lower in Korean people than in those ( $42.5 \pm 5.5$ in males, $33.1 \pm 5.5$ in females) in the New Mexico Aging Process Study [9]. The differences between the SMI reference values may be closely related to differences in ethnicity. In this study, the Korean population showed negative correlations of ASM and SMI; and positive correlations of BMI with age.

Sarcopenia and obesity in the elderly may interactively potentiate their effects on physical disability, morbidity, and mortality. In cross-sectional [30] and longitudinal analyses [28], subjects with sarcopenic obesity were two or three times more likely to develop instrumental disability than those with lean sarcopenia or non-sarcopenic obesity [31]. Among a few studies on the joint effects of sarcopenia and obesity on physical function, Baumgartner [1] observed that sarcopenic obesity showed an 8-and 11-fold higher risk of having more than three physical disabilities in males and females, respectively, older than 60 years of age. Additionally, in a cross-sectional study, the physical impairment was more prominent in people with sarcopenic obesity than in those with either obesity or lean sarcopenia [32].

Several mechanisms have been suggested for the occurrence of OA associated with obesity and sarcopenia. Batushansky et al. [33] reported that obesity may aggravate knee OA by mechanical stress to joints and metabolic stress of adipose tissue. And Shorter et al. [34] suggested the mechanism that skeletal muscle loss affects joint stability and then loss of mobility leads to gradual degeneration of articular cartilage, by which sarcopenia leads to the progression of knee OA.

In this study, the significance of SMI and BMI in age-related ROA was analyzed compared to the KL grade of knee joints. In the Korean population, the KL grade and severity of JSN in knee ROA showed negative correlations with ASM, SMI, and age; and positive correlations with BMI. Higher KL grade was more frequent in the population with age-related low SMI values and BMI-obesity with lower SMI values than in those with normal
SMI and BMI. Subjects with accompanying knee ROA had lower SMI and higher BMI values than those with non-ROA. Furthermore, bony changes of the knee joints were found to be advanced in association with lower SMI and higher BMI values. The probability of a higher KL grade and the incidence of ROA were increased in the population with lower SMI and higher BMI values. Conversely, knees with higher SMI and lower BMI values showed a higher probability of being lower KL grade. The results suggest that low SMI and BMI-defined obesity affected the presence of knee ROA and the severity of JSN in association with aging. BMI showed a consistent increase and correlation with the KL grade and the severity of JSN in knee ROA. And the probability of severe JSN was significantly increased by the presence of obesity. However, the effect of obesity on the severity of knee JSN was not consistent in the case of normal SMI values.

Some studies have reported the correlation of sarcopenia, sarcopenic obesity, and BMI with functional impairment, physical disability, and performance. Lee et al. [35] observed the contribution of BMI-defined sarcopenic obesity to ROA, demonstrating no significant effect of lean sarcopenia on ROA. And Visser et al. [36] reported that a high fat mass/SMM ratio seems to be unfavorable in knee OA.

This study demonstrated that age-related low SMI values might be related to the severity of knee ROA-JSN in the Korean population. However, there were some limitations to this study. The KNHANES data were obtained by a cross-sectional design that did not clearly show the cause and effect of each factor. And further investigations into the effect of sarcopenia on ROA and JSN are needed [37]. As for sarcopenia, muscle strength or physical performance should be considered [5], and both the quantity and quality of skeletal muscle should be evaluated [6].

Taken together, in the Korean population, low SMI and increasing BMI values with aging were correlated with the presence and severity of knee ROA. The results may suggest that the presence and degree of low SMI values are critical to ROA-JSN in obesity. The results of this study demonstrated that maintaining a balanced body composition by securing adequate SMM against aging can be the fundamental and most important strategy in preventing the development and progression of age-related $\mathrm{OA}$.

\section{Notes}

\section{Conflicts of interest}

No potential conflict of interest relevant to this article was reported. 


\section{Funding}

None.

\section{Author contributions}

Conceptualization, Investigation, Data curation, Formal analysis, Funding acquisition, Visualization, Validation: HK, YHH; Methodology, Project administration, Supervision: YHH; Writing-original draft: HK, YHH; Writing-review \& editing: HK, YHH.

\section{ORCID}

Hyun-Je Kim, https://orcid.org/0000-0003-1075-5645

Young-Hoon Hong, https://orcid.org/0000-0001-8119-0464

\section{References}

1. Baumgartner RN. Body composition in healthy aging. Ann N Y Acad Sci 2000;904:437-48.

2. Baumgartner RN, Koehler KM, Gallagher D, Romero L, Heymsfield SB, Ross RR, et al. Epidemiology of sarcopenia among the elderly in New Mexico. Am J Epidemiol 1998;147: 755-63.

3. Kiebzak GM. Age-related bone changes. Exp Gerontol 1991; 26:171-87.

4. Janssen I, Heymsfield SB, Ross R. Low relative skeletal muscle mass (sarcopenia) in older persons is associated with functional impairment and physical disability.J Am Geriatr Soc 2002;50: 889-96.

5. Cruz-Jentoft AJ, Baeyens JP, Bauer JM, Boirie Y, Cederholm T, Landi F, et al. Sarcopenia: European consensus on definition and diagnosis: Report of the European Working Group on Sarcopenia in Older People. Age Ageing 2010;39:412-23.

6. Chen LK, Woo J, Assantachai P, Auyeung TW, Chou MY, Iijima K, et al. Asian Working Group for Sarcopenia: 2019 Consensus Update on Sarcopenia Diagnosis and Treatment. J Am Med Dir Assoc 2020;21:300-7.

7. Hangartner TN, Warner S, Braillon P, Jankowski L, Shepherd J. The Official Positions of the International Society for Clinical Densitometry: acquisition of dual-energy X-ray absorptiometry body composition and considerations regarding analysis and repeatability of measures. J Clin Densitom 2013;16:520-36.

8. Mathus-Vliegen EM. Obesity and the elderly. J Clin Gastroenterol 2012;46:533-44.

9. Pi-Sunyer FX. Obesity: criteria and classification. Proc Nutr Soc 2000;59:505-9.

10. Berrington de Gonzalez A, Hartge P, Cerhan JR, Flint AJ, Hannan L, MacInnis RJ, et al. Body-mass index and mortality among 1.46 million white adults. N Engl J Med 2010;363:
2211-9.

11. WHO Expert Consultation. Appropriate body-mass index for Asian populations and its implications for policy and intervention strategies. Lancet 2004;363:157-63.

12. Loeser RF. Aging processes and the development of osteoarthritis. Curr Opin Rheumatol 2013;25:108-13.

13. Bronson W, Strauss E, Bronson M. Osteoarthritis: eliminating pain and restoring function: an orthopedic perspective. Clin Geriatr 2010;18:9-10.

14. Villareal DT, Apovian CM, Kushner RF, Klein S; American Society for Nutrition; NAASO, The Obesity Society. Obesity in older adults: technical review and position statement of the American Society for Nutrition and NAASO, The Obesity Society. Obes Res 2005; 13:1849-63.

15. Guerne PA, Blanco F, Kaelin A, Desgeorges A, Lotz M. Growth factor responsiveness of human articular chondrocytes in aging and development. Arthritis Rheum 1995;38:960-8.

16. Martin JA, Buckwalter JA. Telomere erosion and senescence in human articular cartilage chondrocytes. J Gerontol A Biol Sci Med Sci 2001;56:B172-9.

17. Tonge DP, Bardsley RG, Parr T, Maciewicz RA, Jones SW. Evidence of changes to skeletal muscle contractile properties during the initiation of disease in the ageing guinea pig model of osteoarthritis. Longev Healthspan 2013;2:15.

18. Barry BK, Sturnieks DL. How important are perturbation responses and joint proprioception to knee osteoarthritis? J Appl Physiol (1985) 2014;116:1-2.

19. Oyeyemi AL. Body mass index, pain and function in individuals with knee osteoarthritis. Niger Med J 2013;54:230-5.

20. Altman R, Asch E, Bloch D, Bole G, Borenstein D, Brandt K, et al. Development of criteria for the classification and reporting of osteoarthritis. Classification of osteoarthritis of the knee. Diagnostic and Therapeutic Criteria Committee of the American Rheumatism Association. Arthritis Rheum 1986;29:1039-49.

21. Kellgren JH, Lawrence JS. Radiological assessment of osteo-arthrosis. Ann Rheum Dis 1957;16:494-502.

22. St-Onge MP. Relationship between body composition changes and changes in physical function and metabolic risk factors in aging. Curr Opin Clin Nutr Metab Care 2005;8:523-8.

23. Heymsfield SB, Smith R, Aulet M, Bensen B, Lichtman S, Wang J, et al. Appendicular skeletal muscle mass: measurement by dual-photon absorptiometry. Am J Clin Nutr 1990;52:214-8.

24. Fielding RA, Vellas B, Evans WJ, Bhasin S, Morley JE, Newman $A B$, et al. Sarcopenia: an undiagnosed condition in older adults. Current consensus definition: prevalence, etiology, and consequences. International working group on sarcopenia. J Am Med Dir Assoc 2011;12:249-56. 
25. Korea Centers for Disease Control \& Prevention (KCDC). Professional surveyor education and quality control for osteoarthritis examination: the fifth National Health and Nutrition Examination Survey, 2010. Daejeon: KCDC; 2011.

26. Jackson AS, Janssen I, Sui X, Church TS, Blair SN. Longitudinal changes in body composition associated with healthy ageing: men, aged 20-96 years. Br J Nutr 2012; 107:1085-91.

27. Rosenberg IH. Summary comments. Am J Clin Nutr 1989;50: 1231-3.

28. Visser M, Langlois J, Guralnik JM, Cauley JA, Kronmal RA, Robbins J, et al. High body fatness, but not low fat-free mass, predicts disability in older men and women: the Cardiovascular Health Study. Am J Clin Nutr 1998;68:584-90.

29. Zamboni M, Mazzali G, Fantin F, Rossi A, Di Francesco V. Sarcopenic obesity: a new category of obesity in the elderly. Nutr Metab Cardiovasc Dis 2008;18:388-95.

30. Visser M, Harris TB, Langlois J, Hannan MT, Roubenoff R, Felson DT, et al. Body fat and skeletal muscle mass in relation to physical disability in very old men and women of the Framingham Heart Study. J Gerontol A Biol Sci Med Sci 1998;53: M214-21.

31. Baumgartner RN, Wayne SJ, Waters DL, Janssen I, Gallagher D, Morley JE. Sarcopenic obesity predicts instrumental activities of daily living disability in the elderly. Obes Res 2004;12:1995-
2004.

32. Walrand S, Guillet C, Salles J, Cano N, Boirie Y. Physiopathological mechanism of sarcopenia. Clin Geriatr Med 2011;27:36585.

33. Batushansky A, Zhu S, Komaravolu RK, South S, Mehta-D'souza P, Griffin TM. Fundamentals of OA. An initiative of osteoarthritis and cartilage. Chapter 9: Obesity and metabolic factors in OA. Osteoarthritis Cartilage 2022;30:501-15.

34. Shorter E, Sannicandro AJ, Poulet B, Goljanek-Whysall K. Skeletal muscle wasting and its relationship with osteoarthritis: a mini-review of mechanisms and current interventions. Curr Rheumatol Rep 2019;21:40.

35. Lee S, Kim TN, Kim SH. Sarcopenic obesity is more closely associated with knee osteoarthritis than is nonsarcopenic obesity: a cross-sectional study. Arthritis Rheum 2012;64:3947-54.

36. Visser AW, de Mutsert R, Loef M, le Cessie S, den Heijer M, Bloem JL, et al. The role of fat mass and skeletal muscle mass in knee osteoarthritis is different for men and women: the NEO study. Osteoarthritis Cartilage 2014;22:197-202.

37. Studenski SA, Peters KW, Alley DE, Cawthon PM, McLean RR, Harris TB, et al. The FNIH sarcopenia project: rationale, study description, conference recommendations, and final estimates. J Gerontol A Biol Sci Med Sci 2014;69:547-58. 